\title{
The story of one Seyfert: from intriguingly ultrasoft to unremarkably average
}

\author{
R. L. C. Starling ${ }^{1} \dagger$, C. Done ${ }^{2}$ and C. Jin ${ }^{2}$ \\ ${ }^{1}$ University of Leicester, Dept. of Physics and Astronomy, Leicester LE1 7RH, UK \\ email: rlcs1@le.ac.uk \\ ${ }^{2}$ Dept. of Physics, Durham University, Durham DH1 3LE, UK
}

\begin{abstract}
We use 4 broadband SEDs spanning 7 years to study the spectral shape and long term variability of RE J2248-511: an unusual AGN discovered in the extreme-UV by the ROSAT Wide Field Camera. This implies a large ultrasoft X-ray flux, which is typical of narrow-line Seyfert 1s, yet its optical lines are clearly broader than the NLS1 definition allows. Here we show that the continuum SED for the brightest epoch dataset is consistent with the mean SED of a standard quasar, and matches well to that from an XMM-SDSS sample of AGN with $M / M_{\odot} \sim 10^{8}$ and $L / L_{\mathrm{Edd}} \sim 0.2$. All the correlated optical and soft X-ray variability can be due entirely to a major absorption event. The only remarkable aspect of this AGN is that there is no measurable intrinsic X-ray absorption column in the brightest epoch dataset. The observed FUV flux is determined by the combination of this and the fact that the source lies within a local absorption hole. RE J2248-511, which once defied classification, demonstrates that characterisation of such objects requires multi-epoch, multi-wavelength campaigns.
\end{abstract}

\section{Motivation}

AGN spectral shapes are complex and varied. Contributions to the optical-X-ray band can arise from the accretion disc, Comptonisation and reflection. Evidence for a separate soft X-ray component exists across the various AGN types (Done et al. 2012; Jin et al. 2013). Studies have found that Eddington ratio is a main driver of SED shape (e.g., Vasudevan \& Fabian 2009). The narrow-line Seyfert 1 (NLS1) class display very steep soft X-ray spectral slopes, which can be highly variable. Given their lower black hole masses, typically $10^{6}-10^{7} M_{\odot}$, much of the soft X-ray emission may arise in the accretion disc (Done et al. 2012; Jin et al.2012), while this cannot be the case for the higher mass, typically less variable, broad-line Seyfert 1s (BLS1). It is curious then, that AGN exist which appear to lie in the BLS1-NLS1 boundary, and we investigate the origin of the SED shapes for one key example here.

\section{RE J2248-511, observations and modelling}

RE J2248-511 ( $z=0.1$ ) was one of only 14 non-blazar AGN selected in the EUV with ROSAT Wide Field Camera (Pounds et al. 1993). Later FUV observations confirmed it had a very bright Big Blue Bump (Dunn et al. 2008,2010), in line with the steep soft X-ray slope measured with ROSAT PSPC (Puchnarewicz et al. 1995). Its relatively broad emission lines $\left(\geqslant 3600 \mathrm{~km} \mathrm{~s}^{-1}\right)$ and high black hole mass $\left(\sim 10^{8} M_{\odot}\right)$ led to an original classification as a BLS1. It was observed several years later with ASCA, where in the common energy range the spectral slope had hardened dramatically, while optical measurements also confirmed extreme variability, akin to NLS1s (Breeveld et al. 2001, 


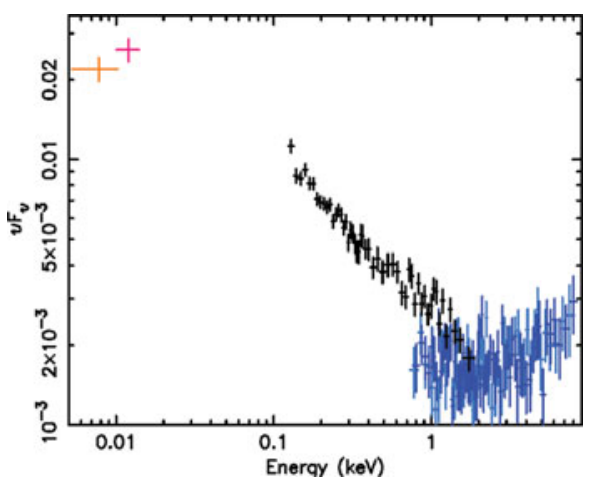

Figure 1. Data prior to our campaign showed a big blue bump + variable X-ray slope. L to R: IUE (1992), FUSE (2002-2004), ROSAT (1993), ASCA (1997).

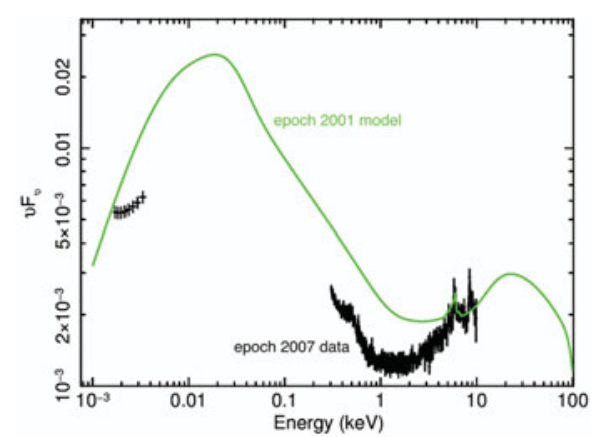

Figure 2. $2007 \mathrm{XMM}+\mathrm{SAAO}$ data showing the departure from the 2001 model, attributable solely to an absorption event.

Fig. 1). We obtained 4 epochs of quasi-simultaneous broadband data over 7 years with XMM+SAAO-1.9m (2000, 2001, 2007) and Swift+Danish Telescope (2006).

The brightest two epochs are well described by a colour temperature corrected accretion disc spectrum with Comptonisation of both low temperature optically thick disc material and high temperature optically thin material in a corona, optxagnf (Done et al. 2012), plus reflection. The best-fitting parameters are typical of an XMM-SDSS $10^{8} M_{\odot}$ broad-line AGN (Jin et al. 2012), in particular $L / L_{\text {Edd }} \sim 0.2$. No absorption whatsoever was required, and the Galactic absorption in that direction is also negligible.

Six years later, the spectrum was dramatically different (Fig. 2). We were able to model this very simply, however, by the addition of a $40 \%$ partial covering neutral absorber. A plausible scenario is that an absorbing cloud (system) with a large column $\left(\sim 5 \times 10^{22}\right.$ $\mathrm{cm}^{-2}$ ) entered our line-of-sight to the AGN between 2005 and 2007. This could indicate a new outflow or mass ejection episode. For full details see Starling et al. (2014).

\section{Summary}

We conclude that RE J2248-511 is an average broad-lined Seyfert 1 galaxy, which appears variable due to transient absorption events. We find no measurable intrinsic $\mathrm{X}$-ray column, and it is seen through a Galactic absorption hole. This low column lineof-sight has given us a unique EUV to X-ray view of an otherwise unremarkable BLS1.

\section{References}

Breeveld, A. A., Puchnarewicz, E. M., \& Otani, C. 2001, MNRAS, 325, 772

Done, C., Davis, S. W., Jin, C., Blaes, O., \& Ward, M. 2012, MNRAS, 420, 1848

Dunn, J. P., Crenshaw, D. M., Kraemer, S. B., \& Trippe, M. L. 2008, AJ, 136, 1201

Dunn, J. P., Crenshaw, D. M., Kraemer, S. B., \& Trippe, M. L. 2010, ApJ, 713, 900

Jin, C., Ward, M., Done, C., \& Gelbord, J. 2012, MNRAS, 420, 1825

Jin, C., Done, C., Middleton, M., \& Ward, M. 2013, MNRAS, 436, 3173

Pounds, K. A. et al. 1993, MNRAS, 260, 77

Puchnarewicz, E., Branduardi-Raymont, G., Mason, K., \& Sekiguchi, K. 1995, MNRAS, 276, 1281

Starling, R. L. C. et al. 2014, MNRAS, 437, 3929

Vasudevan, R. V. \& Fabian, A. C. 2009, MNRAS, 392, 1124 